\section{Kidney \\ Blood Pressure Research}

\title{
Overexpression of Intrarenal Renin- Angiotensin System in Human Acute Tubular Necrosis
}

\author{
Wei Cao Lingwei Jin $^{\mathrm{b}} \quad$ Zhanmei Zhou $^{\mathrm{a}} \quad$ ManqiuYang $^{\mathrm{a}} \quad$ Chunyi Wu ${ }^{\mathrm{a}}$ \\ Liling Wu ${ }^{a}$ Shuang Cui ${ }^{a}$
}

aDivision of Nephrology, Nanfang Hospital, Guangzhou; 'Division of Nephrology, The $2^{\text {nd }}$ Affiliated Hospital \& Yuying Children's Hospital of Wenzhou Medical University, Wenzhou, P.R. China

\section{Key Words}

Acute tubular necrosis $\bullet$ Intrarenal renin-angiotensin system • Urinary angiotensinogen

\begin{abstract}
Background/Aims: Acute tubular necrosis (ATN), a leading cause of acute kidney injury (AKI), is associated with decreased survival and increased progression of chronic kidney disease. A barrier to improving the clinical outcomes is the incomplete understanding of the pathogenesis of AKI. Our objective is to test the hypothesis that intrarenal renin-angiotensin system (RAS) is overexpressed in patients with ATN and could be an indicator of ATN severity. Methods: A transversal study was conducted in patients with biopsy-proven ATN. Intrarenal expression of angiotensinogen and angiotensin II, and urinary angiotensinogen were measured. Results: Patients with ATN demonstrated upregulation of intrarenal RAS, evidenced by upregulation of intrarenal angiotensinogen and angiotensin II. Patients with ATN also have elevated urinary angiotensinogen level that correlated with the overexpressed intrarenal RAS. Moreover, this increase in intrarenal RAS expression and urinary angiotensinogen was associated with the extent of acute tubular injury and urinary albumin excretion, respectively. Conclusions: We demonstrate that the intrarenal RAS is upregulated in patients with ATN and is associated with the severity of ATN. Urinary angiotensinogen reflects intrarenal RAS status, and is of value to assess the severity of ATN.
\end{abstract}

W. Cao, L. Jin and Z. Zhou contributed equally to this work and therefore share first authorship. 


\section{Kidney Blood Pressure Research}

Kidney Blood Press Res 2016;41:746-756

DOI: 10.1159/000450564

Published online: Oktober 28, 2016

(c) 2016 The Author(s). Published by S. Karger AG, Base

Cao et al.: Intrarenal RAS in Human ATN

\section{Introduction}

Acute kidney injury (AKI) is an increasing global concern and imposes an enormous socioeconomic burden [1]. Acute tubular necrosis (ATN), arising as a consequence of septic, toxic, or ischemic insult, is the leading cause of AKI in hospitalized patients [2]. In hospital and intensive care units, ATN accounts for approximate $40 \%$ and $80 \%$ cases of AKI respectively [3], and is associated with decreased survival and increased progression of chronic kidney disease (CKD) [4].

A barrier to improving the clinical outcomes is the incomplete understanding of the pathogenesis of AKI. Several mechanisms have been proposed to contribute to the progression of AKI, including increased inflammation [5, 6] and oxidative stress [7, 8]. Another potential factor is renin-angiotensin system (RAS), which is of great importance for hemodynamic regulation and progression of kidney diseases [9]. Animals with ischemic AKI have shown an increase in intrarenal levels of angiotensin II (Ang II) as compared with sham-operated mice $[10,11]$. Blockade of RAS markedly decelerates renal deterioration in animals with ischemia-reperfusion injury [12-14]. Moreover, angiotensinogen (AGT), the substrate of Ang II, has been identified in proximal tubule and can be secreted to the tubular lumen [15]. Urinary AGT reflects the intrarenal RAS status in CKD [16], and is associated with severity of chronic kidney injury [17]. Recently, several studies found that urinary AGT has been proposed as a predictive and prognostic biomarker for AKI $[18,19]$. These indicate a role for RAS in AKI. However, the exact status of circulating and intrarenal RAS and their relevance to urinary AGT in the pathobiology of human ATN have not been elucidated.

We conducted a transversal study in 30 patients with biopsy-proven ATN. Our objective is to test the hypothesis that the expression of intrarenal RAS, reflected by urinary AGT, is enhanced in patients with ATN and correlated with the severity of ATN. Qualification of urinary AGT could be used to assess the severity of ATN. This is the first study in patients with ATN to demonstrate the upregulation of intrarenal RAS and the utility of urinary AGT as indicator of ATN severity.

\section{Subjects and Methods}

\section{Patients}

This study is approved by Ethical Review Board of the $2^{\text {nd }}$ Affiliated Hospital \& Yuying Children's Hospital, Wenzhou Medical University (Wenzhou, China). All subjects in this study gave written informed consent. Thirty patients, between September 2012 and September 2015, were enrolled according to the inclusion and exclusion criteria described below.

Eligible participants were patients with biopsy-proven ATN aged 18-80 years. Exclusion criteria included: those with preexisting chronic kidney disease (CKD), those with kidney transplantation, and those with comorbid diseases or overlap syndromes such as diabetes, hypertension, liver disease, malignancies and infections. Baseline Serum creatinine was defined as the lowest serum creatinine value in the last 6 months before AKI or, for those without this measurement, the lowest value achieved during hospitalization in the absence of dialysis. CKD was defined as presence of kidney damage (i.e., pathologic abnormalities, abnormalities in urine or imaging tests) or estimated glomerular filtration rate (eGFR) by CKD Epidemiology Collaboration (CKD-EPI) equation $<60 \mathrm{ml} / \mathrm{min}$ per $1.73 \mathrm{~m}^{2}$ for $\geq 3$ months.

\section{Collection of Serum and Urine Samples}

Blood samples were collected at bed rest early in the morning and fresh urine samples were collected after waking up, at the day before the biopsy as described previously [16]. Paired blood and urine samples from sex-and age-matched 21 healthy volunteers were taken as a control. The serum and urine samples were centrifuged at $2500 \mathrm{~g}$ for 10 minutes and the supernatants were stored at $-80^{\circ} \mathrm{C}$. 


\section{Kidney \\ Blood Pressure Research}

Siemens, Germany) and expressed as milligram per milligram of creatinine. The eGFR was determined by the CKD-EPI) equation.

\section{Renal Histological analysis}

Sections from renal biopsy samples were cut at 1-micrometer, and then processed for Hematoxylin and Eosin (HE), Masson's trichrome (MT), Periodic Acid-Schiff (PAS) and Periodic Schiff-Methenamine Silver (PASM) staining. Acute tubular injury was semi-quantitatively assessed by determining the degree of renal tubular necrosis (tubular dilatation, thinning of the tubular epithelium, or cellular casts) using a grading scale of 0 to 4, as described previously with minor modification [20]. Briefly, digital images (at 1360×1024 pixel resolution) of all microscopic fields in each slide were captured at $200 \times$ magnification by the DP 71 CCD camera (Olympus) coupled to a microscope (Olympus, AX-70). The percentage of cortical area affected by ATN was calculated in each field by Image-Pro Plus software (version 6.0, Media Cybernetics). A score was attributed as follows: $1:<25 \%$ of the field affected; $2: 25-50 \%$ of the field affected; $3: 50-75 \%$ of the field affected. 4: > 75\% of the field affected. Data was expressed as the average score in each slide.

To further confirm the accuracy of the semi-quantitative grading of acute tubular injury, morphometry analysis was performed to quantitate the acute tubular injury in each slide [20]. Digital images of all microscopic fields in each slide were captured at $200 \times$ magnification as described above. Cortical areas affected by ATN were measured by Image-Pro Plus software (version 6.0, Media Cybernetics). The results are expressed as percentage of the cortical area affected by ATN in each slide. We found a significant correlation between acute tubular injury score and percentage of the cortical area affected by ATN (data not shown, $\mathrm{r}$ $=0.92, P<0.001$.

\section{Renal Immunohistochemical Analysis of AGT and Ang II}

Renal biopsy samples were obtained from patients with ATN (n=30). Normal kidney tissue adjacent to renal carcinoma obtained from nephrectomy was used as control $(\mathrm{n}=3)$. Two-micrometer-thick sections were processed using an anti-human AGT antibody (1:200, IBL) or anti-human Ang II antibody (1:400, Peninsula Laboratories). Intrarenal expression of AGT and Ang II was quantitated as described previously [21]. Briefly, digital images (at 1360×1024 pixel resolution) of 10 randomly selected tubulointerstitial areas were captured at $400 \times$ magnification by the DP 71 CCD camera (Olympus) coupled to an Olympus AX-70 microscope (Olympus). The staining scores were calculated by Image-Pro Plus software (version 6.0, Media Cybernetics). Data were expressed as the ratio of integrated optical density (IOD) to observed area (IOD/ area), representing the relative intrarenal expression level of AGT and Ang II.

All histological analyses were performed by two pathologists who were blinded to the identity of the tissue. The interobserver reproducibility for semi-quantative histological analyses showed a substantial degree of agreement $(\mathrm{kappa}=0.65)$. To diminish inter-observer discrepancy, slides that received different assessments from the two pathologists were jointly reviewed under a multi-headed microscope and consensus assessment was determined.

\section{ELISA for AGT Quantification}

Concentrations of AGT in serum and urine were detected by an ELISA kit according to the manufacturer's protocol (IBL International, Japan). Values for intra- and inter-assay variability were $3.2 \%$ and $6.3 \%$, respectively. AGT in urine samples were standardized for urinary creatinine and expressed as nanogram per milligram of creatinine.

\section{Statistic Analysis}

Statistical analysis was conducted using the SPSS 17.0 for Windows (SPSS, Chicago, IL). ShapiroWilk goodness of fit test was used for distribution and variance homogeneity. Parameters with a normal distribution are presented as mean $\pm \mathrm{SD}$, and parameters with non-normal distribution are presented as median and interquartile range. Differences in the measured parameters between groups were analyzed by the Student's t-test for normally distributed data or the Mann-Whitney U test for non-normally distributed data. Similarly, correlations were determined using the Pearson's correlation coefficient or the Spearman's rank correlation coefficient depending on the distribution of data. The association between ATN severity and urinary AGT was further confirmed using multiple linear regression analyses by controlling the effect of clinically important confounding variables, such as age, body mass index (BMI), and eGFR at the time of biopsy. In these analyses, urinary AGT was logarithmically transformed to correct for dispersion of data. 


\section{Kidney \\ Blood Pressure Research}

Table 1. Demographic and clinical characteristics

\begin{tabular}{lcccc}
\hline & $\begin{array}{c}\text { healthy volunteers } \\
(\mathrm{n}=21)\end{array}$ & $\begin{array}{c}\text { ATN } \\
(\mathrm{n}=30)\end{array}$ & $t / z$ & $P$ \\
\hline Characteristics at baseline & & & & \\
\hline Age (years) & $32(26-42)$ & $42(26-50)$ & -0.68 & 0.496 \\
Men & $11(52 \%)$ & $22(73 \%)$ & -1.526 & 0.127 \\
Body Mass Index $\left(\mathrm{Kg} / \mathrm{m}^{2}\right)$ & $21.3 \pm 2.67$ & $22.8 \pm 3.41$ & -1.613 & 0.114 \\
Baseline Serum cretinine (mg/dl) & $0.49 \pm 0.11$ & $0.56 \pm 0.13$ & -1.699 & 0.100 \\
\hline Characteristics at the time of biopsy & \multicolumn{5}{c}{} & $/$ & $/$ \\
\hline Days from admission to biopsy (day) & $/$ & $3(2-5)$ & $/$ \\
Serum creatinine $(\mathrm{mg} / \mathrm{dl})$ & $/$ & $4.18 \pm 2.43$ & $/$ \\
eGFR (ml/min/1.73m $\left.{ }^{2}\right)$ & $/$ & $17.95(7.60-41.33)$ & $/$ & $/$ \\
UAE (mg/mg creatinine) & $/$ & $1.150(0.423-2.073)$ & $/$ & $/$ \\
\hline
\end{tabular}

Continuous variables are expressed as the mean \pm SD or median (25th percentile to 75 th percentile [interquartile range]). Categorical variables are expressed as $\mathrm{n}(\%)$. Baseline serum creatinine was defined as the lowest serum creatinine value in the last 6months before AKI or, for those without this measurement, the lowest value achieved during hospitalization in the absence of dialysis. ATN, acute tubular necrosis; eGFR, estimated glomerular filtration rate; UAE, urinary albumin excretion.

The interobserver reproducibility of semi-quantative histological analyses was determined using Cohen's kappa (k) statistics. A two-sided $P<0.05$ was considered statistically significant.

\section{Results}

\section{Patient Characteristics}

Demographics and laboratory data of patients with ATN and healthy volunteers were illustrated in Table 1. Profiles including age, gender, body mass index, and serum creatinine at baseline were comparable among groups.

All the ATN patients had stage 3 AKI according to the 2012 KDIGO clinical practice guideline for AKI. Characteristics of patients with ATN at the time of renal biopsy were demonstrated in Table 1 . The median (interquartile range) time between admission and kidney biopsy was 3 days (interquartile range 2-5 days). None of the patients was being treated with RAS inhibitors at the time of the biopsy.

Overexpression of Intrarenal RAS in Patients with ATN

Acute tubular injury was assessed by PAS staining in renal biopsy samples from patients with ATN. The representative photos were shown in Figure 1a.

Intrarenal expression of AGT and Ang II was evaluated by immunohistochemical staining in renal biopsy samples from patients with ATN and in normal kidney tissue from patients with renal cell carcinoma. As shown in Figure 1b and c, immunoreactivity of AGT was significantly increased in kidney tissue from patients with ATN compared to that in normal kidney tissue $(1.4 \pm 0.6$ versus $0.3 \pm 0.1, P=0.010)$. In patients with ATN, expression of AGT was detected in proximal tubules and distal tubules. However, expression of AGT in normal kidney tissue was observed mainly in proximal tubules.

Immunoreactivity of Ang II was also significantly increased in kidney tissue from patients with ATN compared to normal kidney tissue $(1.1 \pm 0.6$ versus $0.4 \pm 0.1, P=0.021)$ (Figure $1 \mathrm{~d}$ and e). In patients with ATN, expression of Ang II was detected in distal tubules, proximal tubules, and glomerulus. However, expression of Ang II in normal kidney tissue was observed mainly in distal tubules. Collectively, these results suggest that intrarenal RAS is overexpressed in patients with ATN. 


\section{Kidney Blood Pressure Research}

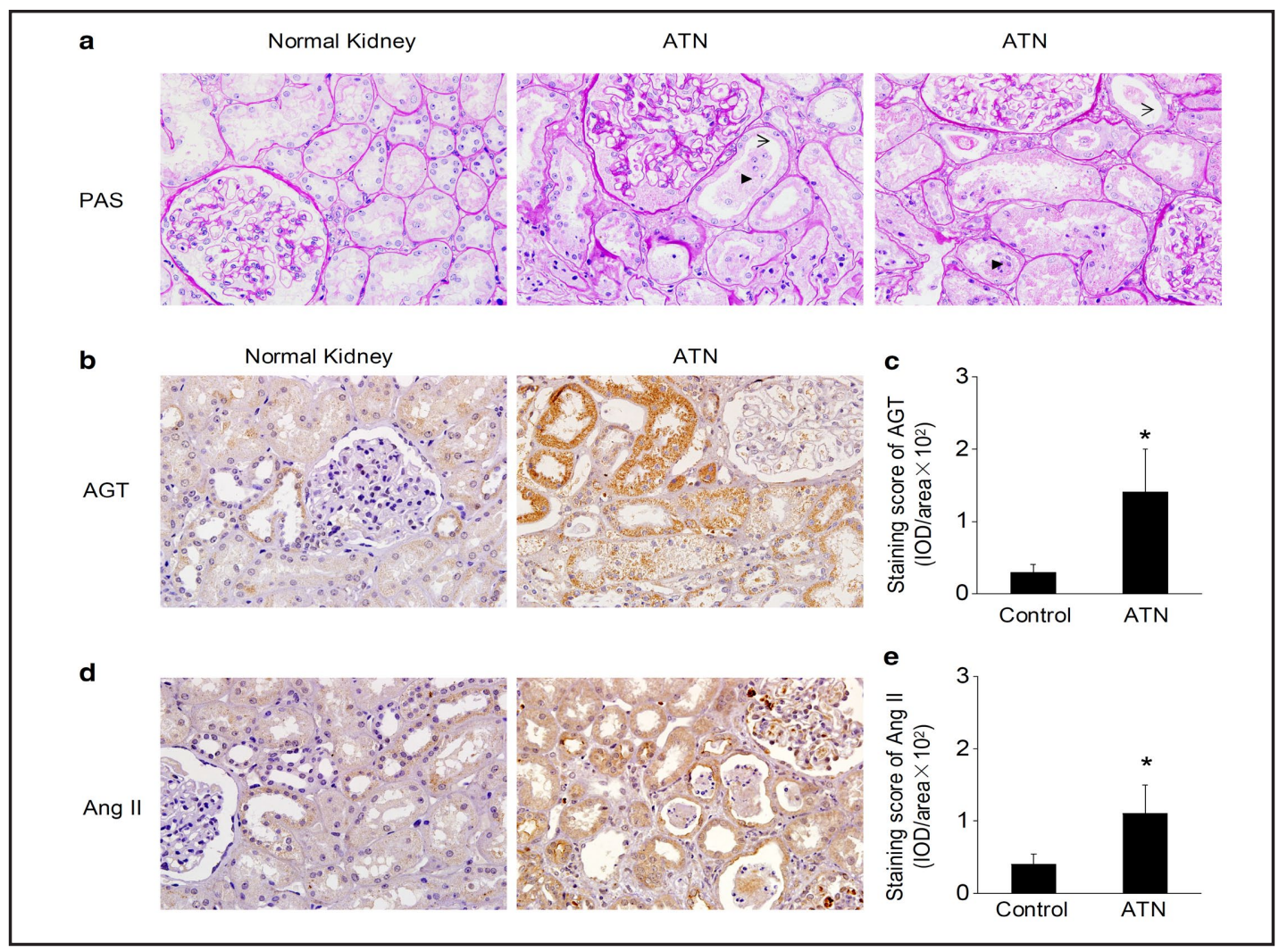

Fig. 1. Upregulation of intrarenal RAS in patients with ATN. (a) Representative photos of acute tubular injury showed by Periodic Acid-Schiff (PAS) staining: tubular dilatation, thinning of the tubular epithelium (arrow), or cellular casts (arrowhead). (b) Representative photos of intrarenal angiotensinogen (AGT) expression showed by immunohistochemical staining. (c) Semi-quantitative data of intrarenal AGT staining. (d) Representative photos of intrarenal Angiotensin II (Ang II) expression showed by immunohistochemical staining. (d) Semi-quantitative data of intrarenal Ang II staining. $t$-test, ${ }^{*} P<0.05$ vs normal kidney.

Urinary AGT level was significantly higher in patients with ATN than that in healthy controls (Figure 2a, $P=0.002$ ). However, unlike urinary AGT, serum AGT levels were similar among groups (Figure $2 \mathrm{~b}, P=0.163$ ), indicating that the urinary AGT may not be derived from the systemic circulation in patients with ATN.

Urinary AGT Levels as a Marker of Intrarenal RAS status in Patients with ATN

Relationship between intrarenal expression of RAS and urinary AGT levels was evaluated in patients with ATN. Intrarenal expression of AGT was correlated positively with urinary AGT (Figure 2c, $r=0.68, P<0.001$ ). Intrarenal expression of Ang II was also correlated positively with urinary AGT (Figure $2 \mathrm{~d}, \mathrm{r}=0.63, P<0.001$ ). Taken together, intrarenal AGT appears to be the major source of urinary AGT. Urinary AGT could be used as a marker for determining intrarenal RAS status.

\section{Intrarenal RAS Expression as an Indicator of ATN Severity}

Correlation between intrarenal expression of RAS and ATN severity was evaluated in patients with ATN. Intrarenal expression of AGT was associated with acute tubular injury score (Figure $3 \mathrm{a}, \mathrm{r}=0.59, P=0.001$ ), percentage of the cortical area affected by ATN (Figure $3 \mathrm{~b}, \mathrm{r}=0.56, \mathrm{P}=0.002$ ), and urinary albumin excretion rate (Figure $3 \mathrm{c}, \mathrm{r}=0.51, P<0.001$ ). Intrarenal expression of Ang II was also correlated with acute tubular injury score (Figure $3 \mathrm{~d}, \mathrm{r}=0.58, P=0.001$ ), percentage of the cortical area affected by ATN (Figure $3 \mathrm{e}, \mathrm{r}=0.59$, 


\section{Kidney \\ Blood Pressure Research}
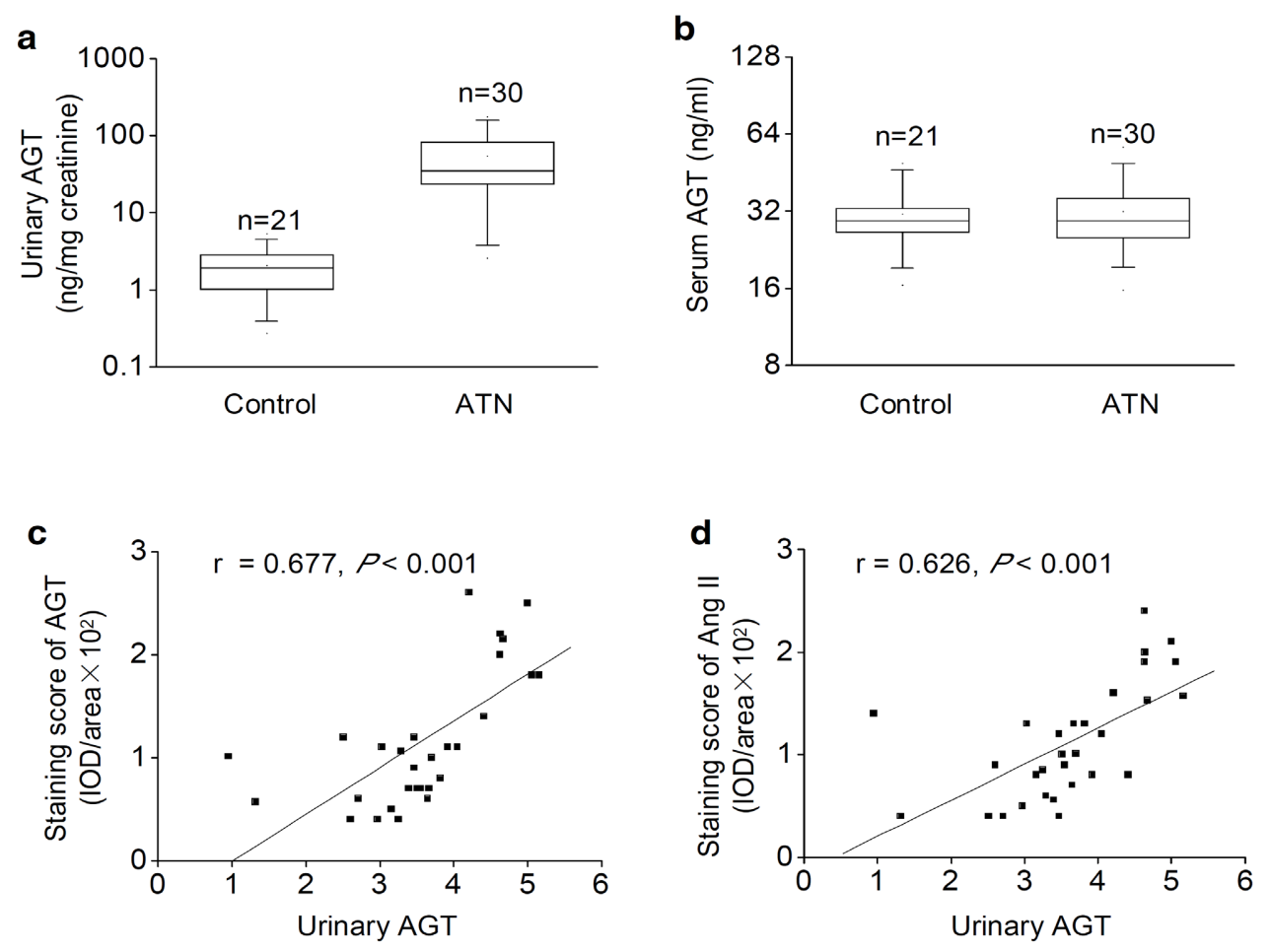

Fig. 2. Expression of intrarenal RAS associated with urinary angiotensinogen level in patients with ATN. (a) Urinary angiotensinogen (AGT) at the time of renal biopsy in patients with ATN and in healthy volunteers (Control). (b) Serum AGT at the time of renal biopsy in patients with ATN and in healthy volunteers. (c) Correlation between intrarenal AGT expression and urinary AGT in patients with ATN. (d) Correlation between intrarenal angiotensin II (Ang II) expression and urinary AGT in patients with ATN. $t$-test, ${ }^{*} P<0.05 v s$ healthy volunteers (control) in a and b. Pearson correlation, ${ }^{*} P<0.05$ in $\mathrm{d}$ and e.

$P<0.001$ ), and urinary albumin excretion rate (Figure $3 f, r=0.53, P=0.004$ ). These results suggest that intrarenal RAS status is associated with the ATN severity.

Urinary AGT as an Indicator of ATN Severity

Correlation between urinary AGT levels and acute kidney injury was determined in patients with ATN. As shown in Figure 4, urinary AGT was associated with acute tubular injury score (Figure $4 \mathrm{a}, \mathrm{r}=0.61, P<0.001$ ) and percentage of the cortical area affected by ATN (Figure $4 \mathrm{~b}, \mathrm{r}=0.62, P<0.001$ ). Urinary AGT was also associated with urinary albumin excretion rate (Figure $4 c, r=0.57, P=0.001$ ). Furthermore, this association between urinary AGT and ATN severity remained statistically significantly after adjustment for age, BMI and eGFR at the time of biopsy (Table 2). All these data further confirm our hypothesis that urinary AGT level could be used as a marker of intrarenal RAS status and could reflect the severity of acute renal injury in patients with ATN.

\section{Discussion}

In the present study, we demonstrate that expression of intrarenal RAS is upregulated in patients with ATN and is associated with the severity of ATN. Urinary angiotensinogen reflects intrarenal RAS status, and could be used as an indicator to evaluate the severity of ATN. This conclusion is supported by several lines of evidence. Firstly, overexpression of intrarenal RAS, evidenced by upregulation of intrarenal AGT and Ang II, was observed in 


\section{Kidney \\ Blood Pressure Research}

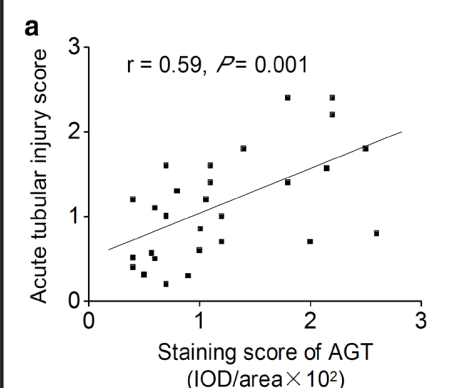

$\left(\right.$ IOD/area $\left.\times 10^{2}\right)$

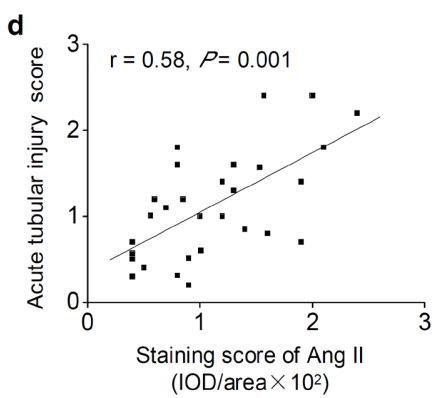

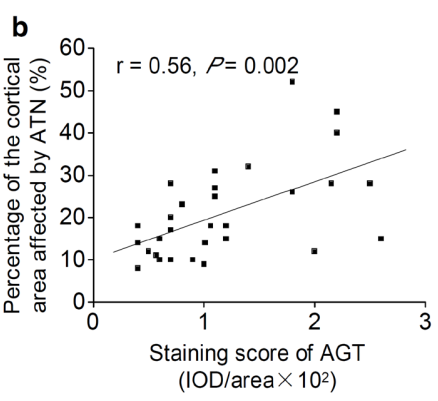
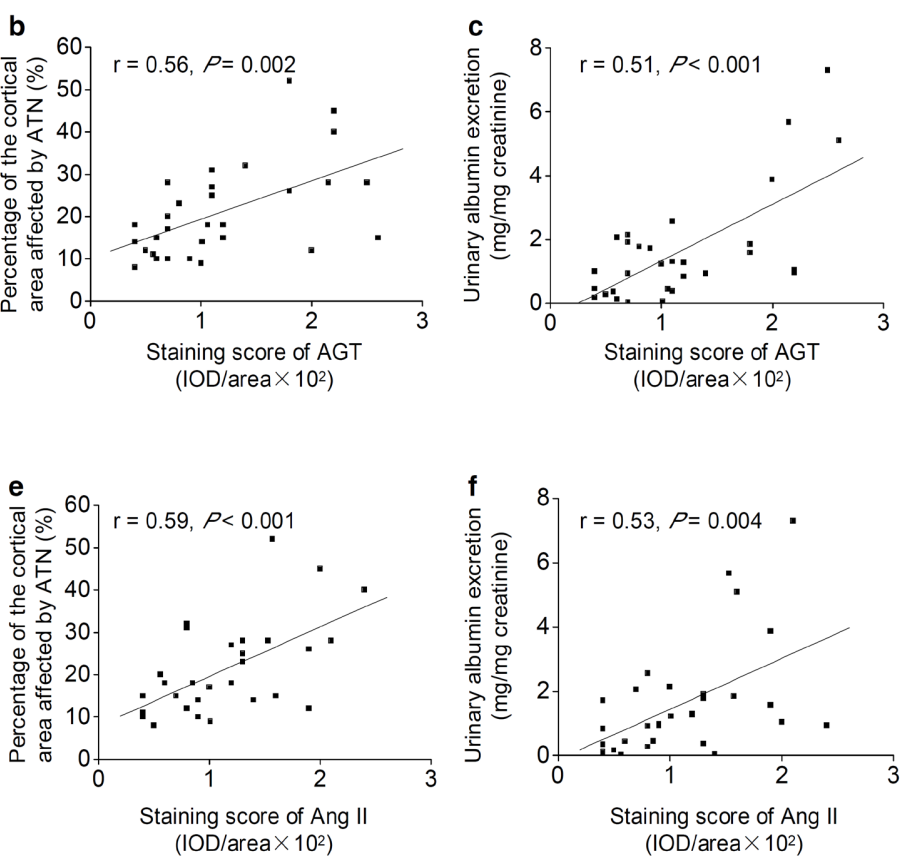

Fig. 3. Intrarenal RAS expression associated with ATN severity. (a) Correlation between intrarenal angiotensinogen (AGT) expression and acute tubular injury score in patients with ATN. (b) Correlation between intrarenal AGT expression and percentage of the cortical area affected by tubular necrosis in patients with ATN. (c) Correlation between intrarenal AGT expression and urinary albumin excretion in patients with ATN. (d) Correlation between intrarenal angiotensin II (Ang II) and acute tubular injury score in patients with ATN. (e) Correlation between intrarenal Ang II expression and percentage of the cortical area affected by tubular necrosis in patients with ATN. (f) Correlation between intrarenal Ang II expression and urinary albumin excretion in patients with ATN. Pearson or Spearman's rank correlation, ${ }^{*} P<0.05$.

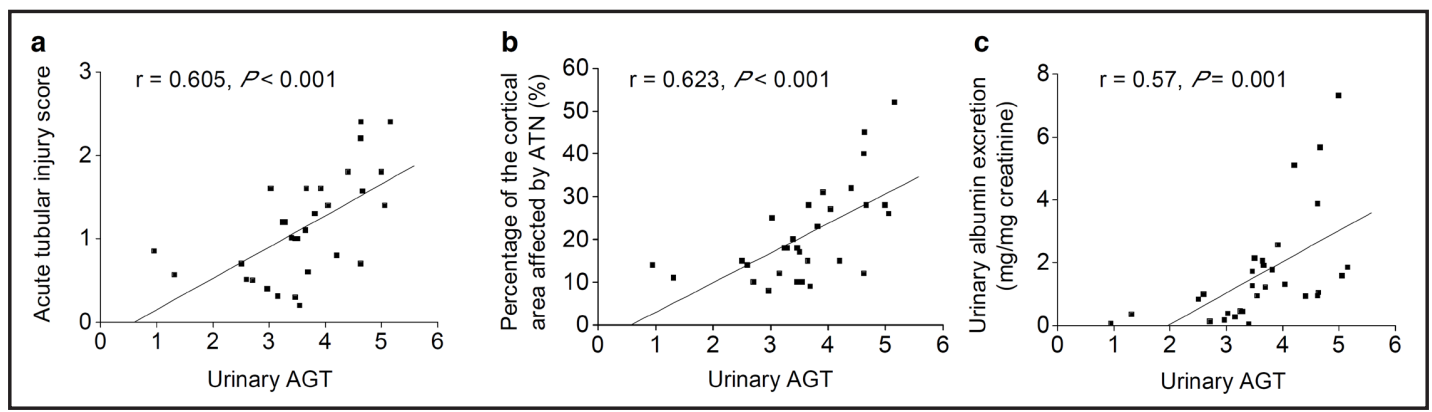

Fig. 4. Urinary angiotensinogen level associated with ATN severity. (a) Correlation between urinary angiotensinogen (AGT) and acute tubular injury score in patients with ATN. (b) Correlation between urinary AGT and percentage of the cortical area affected by tubular necrosis in patients with ATN. (c) Correlation between urinary AGT and urinary albumin excretion in patients with ATN. Pearson or Spearman's rank correlation, ${ }^{*} P<0.05$.

patients with ATN. Secondly, urinary AGT level, which correlated with the increased expression of intrarenal RAS, also elevated in patients with ATN. Moreover, this increase in intrarenal RAS expression and urinary AGT was associated with the extent of acute tubular injury and urinary albumin excretion, respectively. Collectively, these findings indicate that intrarenal RAS status, which is associated with the severity of ATN, can be reflected by urinary AGT. 


\section{Kidney \\ Blood Pressure Research}

Kidney Blood Press Res 2016;41:746-756

DOI: 10.1159/000450564

Published online: Oktober 28, 2016

(C) 2016 The Author(s). Published by S. Karger AG, Base

www.karger.com/kbr

Cao et al.: Intrarenal RAS in Human ATN
Qualification of urinary AGT is of value to assess the ATN severity.

A linkage between

intrarenal RAS and kidney injury has been increasingly recognized. Animal studies have noted an association between inhibition of the RAS and improved outcomes of AKI [22, 23]. Clinical studies have described urinary AGT as a novel predictive and prognostic biomarker of AKI [18, 19]. However, the exact status and relevance of intrarenal RAS
Table 2. Multivariate regression analysis demonstrating urinary AGT is associated with acute tubular injury score in ATN patients ${ }^{\text {a }}$

\begin{tabular}{lccc}
\hline & $\begin{array}{c}\text { Unstandardized } \\
\text { / standardized } \beta\end{array}$ & $t$ & P value \\
\hline Urinary AGT & $0.629 / 0.696$ & 4.237 & $<0.001$ \\
BMI & $-0.046 /-0.13$ & -0.844 & 0.409 \\
eGFR $\left(\mathrm{ml} / \mathrm{min} / 1.73 \mathrm{~m}^{2}\right)$ & $0.003 / 0.131$ & 0.836 & 0.413 \\
Age (years) & $-0.002 / 0.007$ & 0.002 & 0.998 \\
\hline
\end{tabular}

aThe independent effects of variables on acute tubular injury score were analyzed using multivariate regression analysis by the Enter method. $\mathrm{r}=0.78$, adjusted $\mathrm{R}^{2}=0.563$. AGT, angiotensinogen; BMI, body mass index; eGFR, estimated glomerular filtration rate.

in AKI are still unknown. In this study, we firstly demonstrated that intrarenal RAS was upregulated in patients with ATN. Overexpression of AGT was mainly detected in proximal tubules, while Ang II was mainly detected in distal tubules. The proximal tubular AGT, the precursor of Ang II, appears to be secreted directly into the tubular lumen and reach the distal nephron segments [24]. This suggests that the increased intrarenal Ang II in patients with ATN is likely derived from locally formed and secreted AGT.

Moreover, we explore the relationship between intrarenal RAS and ATN severity. Severity of ATN was determined by extent of acute tubular injury, as well as levels of albuminuria due to decreased tubular albumin reabsorption and increased renal albumin gene transcription [25]. Expression of intrarenal RAS significantly correlated with the markers of acute tubular injury, suggesting that expression of intrarenal RAS could be used as an indicator of ATN severity. Chronic kidney disease [26, 27] and diabetes [28, 29] are other examples of intrarenal RAS status linked to the severity of kidney injury.

Urine and serum AGT were also assessed in this study. We found that urinary AGT levels were higher in patients with ATN than that in healthy subjects. This rise in urinary AGT levels was correlated with increase in expression of intrarenal RAS, suggesting that increased urinary AGT in ATN may be due to increased synthesis in the kidney. Indeed, intrarenal AGT has been identified in structures close to the apical membrane of proximal tubular cells [15], which makes its secretion to the urine possible. In addition, our results showed that serum AGT level did not associated with intrarenal RAS status, suggesting intrarenal RAS is regulated independently from circulating RAS. In consistent with our results, several studies observed increased production of intrarenal Ang II in an ischemic AKI model without changes in circulating Ang II [10]. Likewise, in rats infused with human AGT, human AGT was not detectable in the urine because of limited glomerular permeability and/or tubular degradation [30]. These findings support the concept that intrarenal AGT, regulated separately from circulating AGT, is the primary source of urinary AGT in patients with ATN. Urinary AGT could be a powerful tool for determining intrarenal RAS status in these patients. This extends similar findings in patients with chronic kidney disease [31, 32] and hypertension $[30,33]$.

Another new important finding is that urinary AGT can be used as a novel biomarker of the ATN severity. Urinary AGT, which reflect the status of intrarenal RAS, was associated with the extent of acute tubular injury after controlling the effect of clinically important confounding variables [34-36], such as age, BMI and eGFR at the time of biopsy. Increasing evidence suggest that severity of AKI appear to be closely correlated with poor short-term and long-term outcomes [37]. Thus, identifying the severity of AKI early in patients with ATN will help to begin timely intervention and improve their outcomes. Recent studies has yielded several potential biomarkers which correlated with the clinical course of AKI, including neutrophil gelatinase-associated lipocalin [38], kidney injury molecule-1 [39], interleukin-18 [40], and liver fatty acid binding protein [41]. Urinary AGT could potentially be added to these biomarkers to form a biomarker panel, and would probably be particularly 


\section{Kidney \\ Blood Pressure Research}

helpful in serving as a mean to determine intrarenal RAS status and ATN severity. Further clinical investigations with various AKI populations are warranted to confirm the role of urinary AGT as a biomarker of ATN severity.

Given the aforementioned data, we speculate that intrarenal RAS may be linked to the renal injury in AKI, and patients with AKI could potentially benefit from RAS blockade. Indeed, a reduction in GFR, which may be induced by administration of RAS blockers, is not equivalent to more severe renal injury [42]. RAS blockers are not associated with an increase in renal injury in patients with AKI [43]. Although further prospective studies that investigate the therapeutic role of RAS inhibition in patients with AKI are needed, the present study provides direct evidence that overexpression of intrarenal RAS was correlated with the acute renal injury.

This study has several limitations. Since none of the ATN patients in this study were being treated with RAS inhibitors at the time of biopsy, the effect of RAS blockade on urinary AGT and ATN severity was unknown. However, animal studies have already shown a correlation between RAS blockade and improved AKI outcomes [22, 23]. Further studies to investigate the role of RAS inhibition in various AKI populations would be helpful to extend our observations, and to confirm the role of intrarenal RAS in the pathogenesis of AKI. In addition, we did not measure the expression of RAS in infiltrated inflammatory cells. Thus, we cannot exclude the possibility that infiltrated inflammatory cells may generate Ang II and contribute to the progression of renal damage $[44,45]$. However, as presented in our data, the increased intrarenal RAS in ATN patients is likely mainly derived from locally formed and secreted AGT in tubular cells.

\section{Conclusions}

In the present study, we demonstrate that intrarenal RAS is overexpressed in patients with ATN and correlated with the ATN severity. Urinary AGT reflects intrarenal RAS status, and is of value to assess the severity of ATN. This identifies a role for intrarenal RAS in AKI, and thereby may provide supportive evidence for pharmacologic inhibition of the RAS in the treatment of AKI.

\section{Disclosure Statement}

The authors of this manuscript state that they do not have any conflict of interests and nothing to disclose.

\section{Acknowledgements}

Dr. Wei Cao was supported by the National Natural Science Foundation of China (No. 81570619) and Outstanding PhD Thesis Fund Project of Guangdong Province (No. SYBZZXM201322).

\section{References}

1 Lameire NH, Bagga A, Cruz D, De Maeseneer J, Endre Z, Kellum JA, Liu KD, Mehta RL, Pannu N, Van Biesen W, Vanholder R: Acute kidney injury: an increasing global concern. Lancet 2013;382:170-179.

2 Ponte B, Felipe C, Muriel A, Tenorio MT, Liano F: Long-term functional evolution after an acute kidney injury: a 10-year study. Nephrol Dial Transplant 2008;23:3859-3866.

3 Lameire N, Van Biesen W, Vanholder R: Acute renal failure. Lancet 2005;365:417-430. 


\section{Kidney \\ Blood Pressure Research}

4 Gill N, Nally JV, Jr., Fatica RA: Renal failure secondary to acute tubular necrosis: epidemiology, diagnosis, and management. Chest 2005;128:2847-2863.

5 Furuichi K, Wada T, Iwata Y, Kokubo S, Hara A, Yamahana J, Sugaya T, Iwakura Y, Matsushima K, Asano M, Yokoyama H, Kaneko S: Interleukin-1-dependent sequential chemokine expression and inflammatory cell infiltration in ischemia-reperfusion injury. Crit Care Med 2006;34:2447-2455.

6 Inoue T, Okusa MD: Neuroimmune Control of Acute Kidney Injury and Inflammation. Nephron 2015;131:97-101.

7 Zhang G, Wang Q Zhou Q, Wang R, Xu M, Wang H, Wang L, Wilcox CS, Liu R, Lai EY: Protective Effect of Tempol on Acute Kidney Injury Through PI3K/Akt/Nrf2 Signaling Pathway. Kidney Blood Press Res 2016;41:129-138.

8 Yang RL, Wang XT, Liu DW, Liu SB: Energy and oxygen metabolism disorder during septic acute kidney injury. Kidney Blood Press Res 2014;39:240-251.

9 Velez JC: The importance of the intrarenal renin-angiotensin system. Nat Clin Pract Nephrol 2009;5:89100.

10 Allred AJ, Chappell MC, Ferrario CM, Diz DI: Differential actions of renal ischemic injury on the intrarenal angiotensin system. Am J Physiol Renal Physiol 2000;279:F636-645.

11 da Silveira KD, Pompermayer Bosco KS, Diniz LR, Carmona AK, Cassali GD, Bruna-Romero O, de Sousa LP, Teixeira MM, Santos RA, Simoes e Silva AC, Ribeiro Vieira MA: ACE2-angiotensin-(1-7)-Mas axis in renal ischaemia/reperfusion injury in rats. Clin Sci (Lond) 2010;119:385-394.

12 Wang Z, Liu Y, Han Y, Guan W, Kou X, Fu J, Yang D, Ren H, He D, Zhou L, Zeng C: Protective effects of aliskiren on ischemia-reperfusion-induced renal injury in rats. Eur J Pharmacol 2013;718:160-166.

13 Efrati S, Berman S, Hamad RA, Siman-Tov Y, Ilgiyaev E, Maslyakov I, Weissgarten J: Effect of captopril treatment on recuperation from ischemia/reperfusion-induced acute renal injury. Nephrol Dial Transplant 2012;27:136-145.

14 Molinas SM, Cortes-Gonzalez C, Gonzalez-Bobadilla Y, Monasterolo LA, Cruz C, Elias MM, Bobadilla NA, Trumper L: Effects of losartan pretreatment in an experimental model of ischemic acute kidney injury. Nephron Exp Nephrol 2009;112:e10-19.

15 Darby IA, Congiu M, Fernley RT, Sernia C, Coghlan JP: Cellular and ultrastructural location of angiotensinogen in rat and sheep kidney. Kidney Int 1994;46:1557-1560.

16 Yamamoto T, Nakagawa T, Suzuki H, Ohashi N, Fukasawa H, Fujigaki Y, Kato A, Nakamura Y, Suzuki F, Hishida A: Urinary angiotensinogen as a marker of intrarenal angiotensin II activity associated with deterioration of renal function in patients with chronic kidney disease. J Am Soc Nephrol 2007;18:15581565.

17 Kobori H, Ohashi N, Katsurada A, Miyata K, Satou R, Saito T, Yamamoto T: Urinary angiotensinogen as a potential biomarker of severity of chronic kidney diseases. J Am Soc Hypertens 2008;2:349-354.

18 Alge JL, Karakala N, Neely BA, Janech MG, Tumlin JA, Chawla LS, Shaw AD, Arthur JM: Urinary angiotensinogen and risk of severe AKI. Clin J Am Soc Nephrol 2013;8:184-193.

19 Yang X, Chen C, Tian J, Zha Y, Xiong Y, Sun Z, Chen P, Li J, Yang T, Ma C, Liu H, Wang X, Hou FF: Urinary Angiotensinogen Level Predicts AKI in Acute Decompensated Heart Failure: A Prospective, Two-Stage Study. J Am Soc Nephrol 2015;26:2032-2041.

20 Tavares MB, Chagas de Almeida Mda C, Martins RT, de Sousa AC, Martinelli R, dos-Santos WL: Acute tubular necrosis and renal failure in patients with glomerular disease. Ren Fail 2012;34:1252-1257.

21 Xavier LL, Viola GG, Ferraz AC, Da Cunha C, Deonizio JM, Netto CA, Achaval M: A simple and fast densitometric method for the analysis of tyrosine hydroxylase immunoreactivity in the substantia nigra pars compacta and in the ventral tegmental area. Brain Res Brain Res Protoc 2005;16:58-64.

22 Rodriguez-Romo R, Benitez K, Barrera-Chimal J, Perez-Villalva R, Gomez A, Aguilar-Leon D, Rangel-Santiago JF, Huerta S, Gamba G, Uribe N, Bobadilla NA: AT1 receptor antagonism before ischemia prevents the transition of acute kidney injury to chronic kidney disease. Kidney Int 2016;89:363-373.

23 Kontogiannis J, Burns KD: Role of AT1 angiotensin II receptors in renal ischemic injury. Am J Physiol 1998;274:F79-90.

24 Ingelfinger JR, Zuo WM, Fon EA, Ellison KE, Dzau VJ: In situ hybridization evidence for angiotensinogen messenger RNA in the rat proximal tubule. An hypothesis for the intrarenal renin angiotensin system. J Clin Invest 1990;85:417-423. 


\section{Kidney \\ Blood Pressure Research}

25 Ware LB, Johnson AC, Zager RA: Renal cortical albumin gene induction and urinary albumin excretion in response to acute kidney injury. Am J Physiol Renal Physiol 2011;300:F628-638.

26 Kobori H, Katsurada A, Ozawa Y, Satou R, Miyata K, Hase N, Suzaki Y, Shoji T: Enhanced intrarenal oxidative stress and angiotensinogen in IgA nephropathy patients. Biochem Biophys Res Commun 2007;358:156163.

27 Kobori H, Ozawa Y, Suzaki Y, Prieto-Carrasquero MC, Nishiyama A, Shoji T, Cohen EP, Navar LG: Young Scholars Award Lecture: Intratubular angiotensinogen in hypertension and kidney diseases. Am J Hypertens 2006;19:541-550.

28 Singh R, Singh AK, Leehey DJ: A novel mechanism for angiotensin II formation in streptozotocin-diabetic rat glomeruli. Am J Physiol Renal Physiol 2005;288:F1183-1190.

29 Leehey DJ, Singh AK, Bast JP, Sethupathi P, Singh R: Glomerular renin angiotensin system in streptozotocin diabetic and Zucker diabetic fatty rats. Transl Res 2008;151:208-216.

30 Kobori H, Nishiyama A, Harrison-Bernard LM, Navar LG: Urinary angiotensinogen as an indicator of intrarenal Angiotensin status in hypertension. Hypertension 2003;41:42-49.

31 Kobori H, Nangaku M, Navar LG, Nishiyama A: The intrarenal renin-angiotensin system: from physiology to the pathobiology of hypertension and kidney disease. Pharmacol Rev 2007;59:251-287.

32 Urushihara M, Kondo S, Kagami S, Kobori H: Urinary angiotensinogen accurately reflects intrarenal ReninAngiotensin system activity. Am J Nephrol 2010;31:318-325.

33 Kobori H, Alper AB, Jr., Shenava R, Katsurada A, Saito T, Ohashi N, Urushihara M, Miyata K, Satou R, Hamm LL, Navar LG: Urinary angiotensinogen as a novel biomarker of the intrarenal renin-angiotensin system status in hypertensive patients. Hypertension 2009;53:344-350.

34 Bagshaw SM, George C, Bellomo R: Early acute kidney injury and sepsis: a multicentre evaluation. Crit Care 2008;12:R47.

35 Barrantes F, Tian J, Vazquez R, Amoateng-Adjepong Y, Manthous CA: Acute kidney injury criteria predict outcomes of critically ill patients. Crit Care Med 2008;36:1397-1403.

36 Lechevallier E, Dussol B, Luccioni A, Thirion X, Vacher-Copomat H, Jaber K, Brunet P, Leonetti F, Lavelle O, Coulange C, Berland Y: Posttransplantation acute tubular necrosis: risk factors and implications for graft survival. Am J Kidney Dis 1998;32:984-991.

37 Chawla LS, Amdur RL, Amodeo S, Kimmel PL, Palant CE: The severity of acute kidney injury predicts progression to chronic kidney disease. Kidney Int 2011;79:1361-1369.

38 Mori K, Lee HT, Rapoport D, Drexler IR, Foster K, Yang J, Schmidt-Ott KM, Chen X, Li JY, Weiss S, Mishra J, Cheema FH, Markowitz G, Suganami T, Sawai K, Mukoyama M, Kunis C, D'Agati V, Devarajan P, Barasch J: Endocytic delivery of lipocalin-siderophore-iron complex rescues the kidney from ischemia-reperfusion injury. J Clin Invest 2005;115:610-621.

39 Han WK, Bailly V, Abichandani R, Thadhani R, Bonventre JV: Kidney Injury Molecule-1 (KIM-1): a novel biomarker for human renal proximal tubule injury. Kidney Int 2002;62:237-244.

40 Parikh CR, Jani A, Melnikov VY, Faubel S, Edelstein CL: Urinary interleukin-18 is a marker of human acute tubular necrosis. Am J Kidney Dis 2004;43:405-414.

41 Yamamoto T, Noiri E, Ono Y, Doi K, Negishi K, Kamijo A, Kimura K, Fujita T, Kinukawa T, Taniguchi H, Nakamura K, Goto M, Shinozaki N, Ohshima S, Sugaya T: Renal L-type fatty acid--binding protein in acute ischemic injury. J Am Soc Nephrol 2007;18:2894-2902.

42 Alge JL, Arthur JM: Biomarkers of AKI: a review of mechanistic relevance and potential therapeutic implications. Clin J Am Soc Nephrol 2015;10:147-155.

43 Coca SG, Garg AX, Swaminathan M, Garwood S, Hong K, Thiessen-Philbrook H, Passik C, Koyner JL, Parikh CR: Preoperative angiotensin-converting enzyme inhibitors and angiotensin receptor blocker use and acute kidney injury in patients undergoing cardiac surgery. Nephrol Dial Transplant 2013;28:2787-2799.

44 Nava M, Quiroz Y, Vaziri N, Rodriguez-Iturbe B: Melatonin reduces renal interstitial inflammation and improves hypertension in spontaneously hypertensive rats. Am J Physiol Renal Physiol 2003;284:F447454.

45 Rodriguez-Iturbe B, Zhan CD, Quiroz Y, Sindhu RK, Vaziri ND: Antioxidant-rich diet relieves hypertension and reduces renal immune infiltration in spontaneously hypertensive rats. Hypertension 2003;41:341-346. 